\title{
Large-scale copy number analysis reveals variations in genes not previously associated with malignant pleural mesothelioma
}

\author{
Marieke Hylebos ${ }^{1,2}$, Guy Van Camp ${ }^{1,2}$, Geert Vandeweyer ${ }^{1}$, Erik Fransen ${ }^{1,3}$, Matthias \\ Beyens $^{1,2}$, Robin Cornelissen ${ }^{4}$, Arvid Suls ${ }^{1}$, Patrick Pauwels ${ }^{2,5}$, Jan P. van Meerbeeck ${ }^{2,6}$ \\ and Ken Op de Beeck ${ }^{1,2}$ \\ ${ }^{1}$ Center of Medical Genetics, University of Antwerp and Antwerp University Hospital, 2650 Antwerp, Belgium \\ ${ }^{2}$ Center for Oncological Research, University of Antwerp, 2610 Antwerp, Belgium \\ ${ }^{3}$ StatUa Center for Statistics, University of Antwerp, 2610 Antwerp, Belgium \\ ${ }^{4}$ Department of Pulmonary Medicine, Erasmus Medical Center Cancer Institute, 3015 Rotterdam, The Netherlands \\ ${ }^{5}$ Laboratory of Pathology, Antwerp University Hospital, 2650 Antwerp, Belgium \\ ${ }^{6}$ Thoracic Oncology, Antwerp University Hospital, 2650 Antwerp, Belgium
}

Correspondence to: Marieke Hylebos, email: Marieke.Hylebos@uantwerpen.be

Keywords: malignant pleural mesothelioma; copy number variations; low-pass whole genome sequencing; the cancer genome atlas; cancer census genes

Received: August 01, $2017 \quad$ Accepted: November 15, $2017 \quad$ Published: December 01, 2017

Copyright: Hylebos et al. This is an open-access article distributed under the terms of the Creative Commons Attribution License 3.0 (CC BY 3.0 ), which permits unrestricted use, distribution, and reproduction in any medium, provided the original author and source are credited.

\section{ABSTRACT}

Malignant pleural mesothelioma (MPM) is an aggressive tumor that is often causally associated with asbestos exposure. Comparative genomic hybridization techniques and arrays demonstrated a complex set of copy number variations (CNVs) in the MPM-genome. These techniques however have a limited resolution, throughput and flexibility compared to next-generation sequencing platforms.

In this study, the presence of CNVs in the MPM-genome was investigated using an MPM-cohort $(N=85)$ for which genomic microarray data are available through 'The Cancer Genome Atlas' (TCGA). To validate these results, the genomes of MPMs and matched normal samples $(N=21)$ were analyzed using low-pass whole genome sequencing on an 'Illumina HiSeq' platform. CNVs were detected using in-house developed analysis pipelines and frequencies of copy number loss and gain were calculated.

In both datasets, losses on chromosomes 1, 3, 4, 6, 9, 13 and 22 and gains on chromosomes 1, 5, 7 and 17 were found in at least $25 \%$ and $15 \%$ of MPMs, respectively. Besides the well-known MPM-associated genes, CDKN2A, NF2 and BAP1, other interesting cancer-associated genes were listed as frequently involved in a copy number loss (e.g. EP300, SETD2 and PBRM1). Moreover, four cancer-associated genes showed a high frequency of copy number gain in both datasets (i.e. TERT, FCGR2B, CD79B and PRKAR1A). A statistically significant association between overall survival and the presence of copy number loss in the CDKN2A-containing region was observed in the TCGA-set.

In conclusion, recurrent CNVs were detected in both datasets, occurring in regions harboring known MPM-associated genes and genes not previously linked to MPM.

\section{INTRODUCTION}

Malignant pleural mesothelioma (MPM) is a rare and highly aggressive cancer originating from the mesothelial cells of the pleura [1]. A causal relationship between the development of MPM and exposure to asbestos has been demonstrated, with up to $80 \%$ of all patients being professionally exposed in the 30 to 40 years preceding the diagnosis [2]. Due to differences in historical asbestos import, consumption and ban, the incidence of MPM greatly varies between countries worldwide, ranging from seven patients per million inhabitants in Japan to 40 
patients per million inhabitants in Australia [3]. Moreover, since asbestos is still being used in some non-Western and Western countries, MPM will remain a global health issue for decades to come [4]. Besides this increasing incidence, patients diagnosed with MPM still face a poor prognosis. The median overall survival time of untreated patients is six to ten months with a 5-year survival rate below 5\%. Furthermore, current therapeutic options are limited and seem to provide only modest survival benefit $[5,6]$.

Genetic analyses have revealed genetic alterations in a number of genes in MPM. Of these, somatic inactivation of the tumor suppressor genes $C D K N 2 A, N F 2$ and $B A P 1$ seems to be the most prevalent [7-9]. Additionally, the presence of a complex and heterogeneous set of chromosomal copy number variations (CNVs) in MPM was described. Although no single MPM-specific alteration was observed, losses in chromosomes 1p, 4q, 9p, 13q, 14q and $22 \mathrm{q}$ were commonly noted using karyotype analyses and (microarray-based) comparative genomic hybridization techniques [10-19]. These techniques however have a limited resolution compared to highly sensitive nextgeneration sequencing platforms, which allow genomewide detections in a high-throughput manner.

Here, we investigated the presence of CNVs in the MPM-genome using an MPM-cohort $(N=85)$, for which genomic microarray data are available through 'The Cancer Genome Atlas' (TCGA). These results were validated using low-pass whole genome sequencing (LP-WGS) on genomic DNA from paired tumor and normal samples of 21 MPM-patients. We found recurrent CNVs in several regions, harboring interesting cancer-associated genes.

\section{RESULTS}

\section{The Cancer Genome Atlas}

\section{Copy number variations in MPM}

Segmented copy number data of $85 \mathrm{MPMs}$, available through the TCGA-website, were used to assess the MPM copy number profile (Table 1). In order to identify regions with recurrent CNVs in the $85 \mathrm{MPMs}$, frequencies of copy number loss and gain were calculated using the 'Multi-intersect tool' from 'BEDtools' (Figure 1) [20]. Large losses occurring in more than $25 \%$ of cases were identified on chromosomes 1 (p36.33-p36.13 and p31.1-p13.1), 3 (p22.2-p14.2), 4 (q13.1-q35.2), 6 (q14.1-q27), 9 (p22.2-p21.1), 13 (q11-q22.3), 14 (q11.1-q32.33) and 22 (q11.1-q13.33). Some regions on chromosome 22 were even lost in up to $75 \%$ of all studied MPMs. Gains occurred less frequently, with large regions on chromosomes 1 (q21.2-q44), 5 (p15.33-p11), 7 (p22.3-q11.21 and q11.21-q31.33) and 17 (q21.32-q25.1) exhibiting gains in more than $15 \%$ of MPMs.

In order to identify potentially interesting genes within regions exhibiting recurrent CNVs in the TCGA-dataset, the exact frequency of copy number loss and gain in the regions containing 'Cancer census genes' was listed [21]. The 'top 20' list of 'Cancer census genes' most frequently involved in a copy number loss contained some well-known MPM-associated genes (Table 2). Whereas $N F 2$ was lost in $62 \%$ of cases, $C D K N 2 A$ was lost in $51 \%$ and $B A P 1$ in $44 \%$ of MPMs. However, the list also contained other genes, some even lost in a higher frequency of samples (e.g. EP300, PDGFB, MKL1, MYH9, APOBEC3B and ZNF278). EP300 for example was located in a chromosomal region lost in $69 \%$ of all MPMs, being the highest reported frequency of copy number loss (Table 2). EP300 encodes an histone acetyltransferase, regulating transcription via chromatin remodeling and influencing cell proliferation and differentiation $[22,23]$. The frequency of copy number gain in regions containing 'Cancer census genes' was remarkably lower compared to the frequency of copy number loss (highest frequency of gain: $27 \%$ versus loss: $69 \%$, Table 2). Nevertheless, regions containing some interesting 'Cancer census genes' on chromosomes 5,1 and 17 showed copy number gain in a substantial number of patients. The region containing TERT, the gene encoding the catalytic component of the telomerase enzyme [24], exhibited a copy number gain in up to $27 \%$ of MPMs, being the most frequently reported copy number gain (Table 2 ).

\section{Association with clinical and histological parameters}

Associations between clinicopathological parameters and the presence of copy number loss (segment mean $\leq-0.25$ ) or gain (segment mean $\geq 0.25$ ) in the regions containing the most frequently involved 'Cancer census genes' (Table 2) were investigated. When for a certain sample a gene was spread over multiple segments with different segment means, this sample was not considered when examining potential associations. No statistically significant associations with gender, age at diagnosis (before or after the age of 60), asbestos exposure or histological diagnosis (epithelioid or nonepithelioid) were found (Supplementary Tables 1 and 2, depicting the $p$-values for the investigated associations). However, a statistically significant association was found between a survival less than 36 months and the presence of copy number loss in the segment containing CDKN2A ( $p$-value: 0.01 ). Moreover, a univariate survival analysis showed a significantly longer survival time for patients with tumors without copy number loss in the segment containing $C D K N 2 A$ ( $p$-value: $4.54 \mathrm{e}^{-6}$, median survival of 982 days versus 456 days for patients with tumors with $C D K N 2 A$ loss, Figure 2). Univariate analyses of the prognostic effect of gender, histologic subtype and age at diagnosis were not significant ( $p$-values: $0.446 ; 0.0895$ and 0.382 respectively). A non-significant trend towards an association between age at diagnosis (younger or older than 60 years) and the presence of copy number gain in the segment containing TERT was identified (p-value: 0.07). 
Table 1: Clinical characteristics of the included MPM-patients (TCGA-set and LP-WGS-set)

\begin{tabular}{|c|c|c|}
\hline \multirow{2}{*}{ Patient characteristics } & \multicolumn{2}{|c|}{ Absolute amount (percentage) } \\
\hline & TCGA $(N=85)$ & LP-WGS $(N=21)$ \\
\hline $\begin{array}{l}\text { Gender } \\
\text { Male } \\
\text { Female }\end{array}$ & $\begin{array}{l}69(81 \%) \\
16(19 \%)\end{array}$ & $\begin{array}{l}19(90 \%) \\
2(10 \%)\end{array}$ \\
\hline $\begin{array}{l}\text { Age at diagnosis } \\
\text { Before or at the age of } 60 \\
\text { After the age of } 60 \\
\text { Unknown } \\
\end{array}$ & $\begin{array}{l}32(38 \%) \\
53(62 \%) \\
0(0 \%) \\
\end{array}$ & $\begin{array}{l}9(43 \%) \\
10(48 \%) \\
2(10 \%) \\
\end{array}$ \\
\hline $\begin{array}{l}\text { History of asbestos exposure } \\
\text { Yes } \\
\text { No } \\
\text { Unknown } \\
\end{array}$ & $\begin{array}{l}54(64 \%) \\
14(16 \%) \\
17(20 \%) \\
\end{array}$ & \begin{tabular}{|l|}
$12(57 \%)$ \\
$0(0 \%)$ \\
$9(43 \%)$ \\
\end{tabular} \\
\hline $\begin{array}{l}\text { Histologic diagnosis } \\
\text { Epithelioid mesothelioma } \\
\text { Non-epithelioid mesothelioma } \\
\text { Unknown }\end{array}$ & $\begin{array}{l}56(66 \%) \\
24(28 \%) \\
5(6 \%)\end{array}$ & $\begin{array}{l}18(86 \%) \\
3(14 \%) \\
0(0 \%)\end{array}$ \\
\hline $\begin{array}{l}\text { Platinum/pemetrexed treatment prior to tissue collection } \\
\text { No } \\
\text { Yes }\end{array}$ & $\begin{array}{l}85(100 \%) \\
0(0 \%)\end{array}$ & $\begin{array}{l}10(48 \%) \\
11(52 \%)\end{array}$ \\
\hline $\begin{array}{l}\text { Time to death or last follow-up } \\
\text { Less than } 36 \text { months } \\
\text { More than } 36 \text { months } \\
\text { Unknown } \\
\end{array}$ & $\begin{array}{l}73(86 \%) \\
11(13 \%) \\
1(1 \%) \\
\end{array}$ & \begin{tabular}{|l|}
$16(76 \%)$ \\
$3(14 \%)$ \\
$2(10 \%)$ \\
\end{tabular} \\
\hline $\begin{array}{l}\text { Vital status } \\
\text { Dead } \\
\text { Alive }\end{array}$ & $\begin{array}{l}56(66 \%) \\
29(34 \%)\end{array}$ & $\begin{array}{l}15(71 \%) \\
6(29 \%)\end{array}$ \\
\hline
\end{tabular}

\section{Low-pass whole genome sequencing}

\section{Copy number variations in MPM}

To validate the results we obtained via the TCGAdataset, 21 MPMs and matched normal samples were assessed for CNVs using LP-WGS (Table 1). For this analysis, the genome was divided in $50 \mathrm{~kb}$-bins and for every bin, the $\log _{2}$-ratio comparing tumor versus normal sample was determined (Supplementary Figure 1, depicting the copy number profile of a representative sample pair). As in the data obtained using the TCGA-set, it was observed that copy number losses occurred more frequently in these MPMs compared to copy number gains.

In order to identify regions with recurrent $\mathrm{CNVs}$ in the 21 MPMs, two different approaches were followed. A first strategy was based on calculating the frequencies of both copy number loss and gain in each of the $50 \mathrm{~kb}$-bins. Doing so, regions with recurrent gains and losses were observed (Figure 3). Large losses occurring in more than $25 \%$ of cases were identified on parts of chromosomes 1 (p31.1-p11.2), 3 (p22.3-p14.1), 4 (p16.3-p11 and q12-q35.2), 6 (q15-q27), 9 (p23-p21.1), 13 (q11-q34), 17 (p13.3-p11.2) and 22 (q11.1-q13.33), with some regions being lost in up to $60 \%$ of all MPMs. Gains occurred less frequently, with regions less easy to demarcate on chromosomes 1 (q21.2-q44), 2 (p25.3-p22.3), 3 (q24-q29), 5 (p15.33-p11 and q11.1-q35.3), 7 (p22.3-p11.2 and q11.21-q36.3), 15 (q21.1-q26.3), 17 (q11.2-q25.3), 18 (p11.32-p11.21 and q11.1-q23) and 19 (p13.3-p12 and q11-q13.43) exhibiting gains in more than $15 \%$ of MPMs.

Similar as for the TCGA-data, the exact frequency of copy number gain and loss in the regions containing 'Cancer census genes' was assessed [21]. In contrast to the TCGA-data, the 'top 20' list of 'Cancer census genes' most frequently involved in a copy number loss in the LP-WGS-data did not contain NF2, CDKN2A or BAP1 (Table 3). These genes were lost in $48 \%, 52 \%$ and $43 \%$ of MPMs respectively, which was not enough to rank them in the top. Six other 'Cancer census genes' however, were listed among the most frequently lost ones, both in the TCGA- and LP-WGS-dataset (i.e. EP300, SETD2, PBRM1, CHEK2, MKL1 and MAPK1). As in the TCGAdata, EP300 was the 'Cancer census gene' with the highest reported frequency of copy number loss, being in $71 \%$ of studied MPMs (Table 3). The frequency of copy number gain in regions containing 'Cancer census genes' was in line with that obtained in the TCGA-data and remarkably 
lower compared to the frequency of copy number loss (Table 3). Of the 'Cancer census genes' most frequently involved in a copy number gain, four were in common with those in the TCGA-data (i.e. FCGR2B, TERT, CD79B and PRKAR1A). PMS2, a gene encoding a component of the DNA mismatch repair system [25], was involved in a copy number gain in up to $33 \%$ of MPMs, being the most frequently reported copy number gain (Table 3 ).

A second and novel strategy to determine regions with recurrent CNVs in the 21 MPMs was based on calculating the mean $\log _{2}$-ratio for every $50 \mathrm{~kb}$-bin over the 21 sample pairs (Figure 3). Although this strategy precludes the identification of regions exhibiting both losses and gains in different tumors, these regions are less likely to be important in MPM-tumorigenesis. As a result, a sharper focus on the most interesting regions is obtained. In order to statistically summarize this information, a onesample $t$-test was performed for every $50 \mathrm{~kb}$-bin, with the null hypothesis assuming a mean $\log _{2}$-ratio of 0 . In regions with mean $\log _{2}$-ratios different from $0, p$-values were not uniformly distributed and some even shifted towards the significance threshold. A Kolmogorov-Smirnov (K-S) test was performed to confirm the non-uniform distribution of these $p$-values. Regions in which the $p$-value of the $\mathrm{K}-\mathrm{S}$ test was smaller than $10^{-15}$ were listed in Table 4 . The negative logarithm of the $p$-value of the K-S test was plotted against chromosome position (Supplementary Figure 2, depicting the mean copy number profile over the 21 sample pairs).

\section{Association with clinical and histological parameters}

Associations between clinicopathological parameters and the presence of copy number loss $\left(\log _{2}-\right.$ ratio $\leq-0.25)$ or gain $\left(\log _{2}\right.$-ratio $\left.\geq 0.25\right)$ in the regions containing the most frequently involved 'Cancer census genes’ (Table 3) were tested. No statistically significant

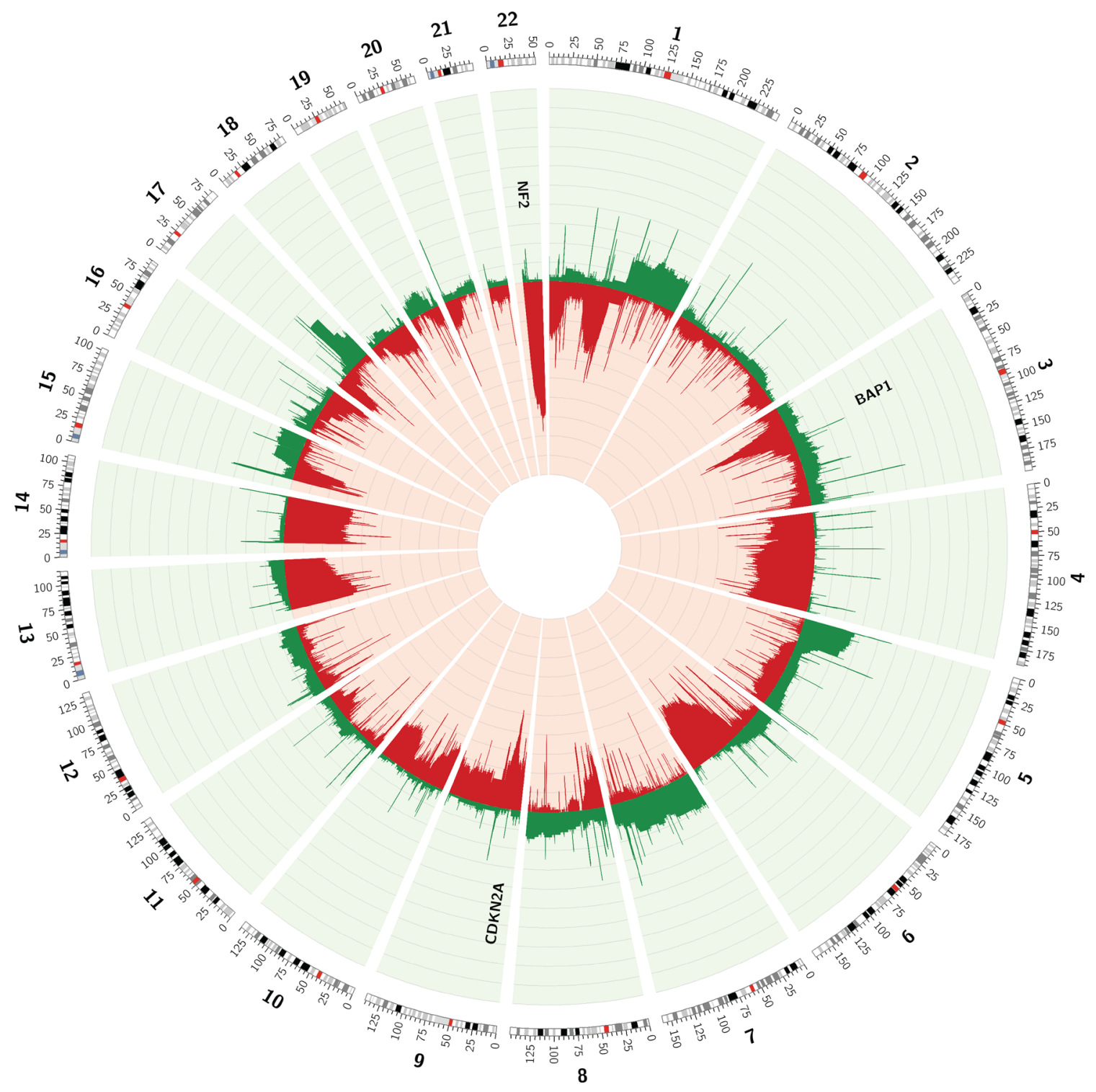

Figure 1: Circos plot of the CNVs observed in array data of 85 MPMs, available through TCGA. Frequencies of copy number loss (red) and gain (green) are depicted for every chromosome position. 
Table 2: 'Cancer census genes' most frequently involved in a copy number loss or gain in the TCGAdata

\begin{tabular}{|c|c|c|c|c|c|c|c|}
\hline \multicolumn{4}{|c|}{$\begin{array}{l}\text { 'Cancer census genes' most frequently involved in a copy } \\
\text { number loss }{ }^{\mathrm{a}}\end{array}$} & \multicolumn{4}{|c|}{$\begin{array}{l}\text { 'Cancer census genes' most frequently involved in a copy } \\
\text { number gain }{ }^{\mathrm{a}}\end{array}$} \\
\hline Gene name & $\begin{array}{l}\text { Chromosome } \\
\text { position }\end{array}$ & $\begin{array}{l}\text { OG or } \\
\text { TS }^{\mathbf{b}}\end{array}$ & $\begin{array}{l}\text { Frequency } \\
\operatorname{loss}(\%)^{c}\end{array}$ & Gene name & $\begin{array}{l}\text { Chromosome } \\
\text { position }\end{array}$ & $\begin{array}{l}\text { OG or } \\
\text { TS }^{\mathbf{b}}\end{array}$ & $\begin{array}{l}\text { Frequency } \\
\text { gain }(\%)^{\mathrm{c}}\end{array}$ \\
\hline EP300 & $\begin{array}{l}\text { chr22:41,488,614- } \\
41,576,081\end{array}$ & / & 69.41 & TERT & $\begin{array}{l}\text { chr5:1,253,287- } \\
1,295,162\end{array}$ & 1 & 27.06 \\
\hline$P D G F B$ & $\begin{array}{l}\text { chr22:39,619,685- } \\
39,640,957\end{array}$ & OG & 68.24 & SDHA & $\begin{array}{l}\text { chr5:218,356- } \\
256,814\end{array}$ & TS & 24.71 \\
\hline$M K L 1$ & $\begin{array}{l}\text { chr22:40,806,292- } \\
41,032,690\end{array}$ & / & 68.24 & DROSHA & $\begin{array}{l}\operatorname{chr} 5: 31,400,602- \\
31,532,282\end{array}$ & TS & 23.53 \\
\hline МYH9 & $\begin{array}{l}\text { chr22:36,677,323- } \\
36,784,063\end{array}$ & 1 & 68.24 & $I L 7 R$ & $\begin{array}{l}\text { chr5:35,856,977- } \\
35,879,705\end{array}$ & 1 & 22.35 \\
\hline АРОВЕС $3 B$ & $\begin{array}{l}\text { chr22:39,378,404- } \\
39,388,784\end{array}$ & OG/TS & 64.71 & LIFR & $\begin{array}{l}\text { chr5:38,475,065- } \\
38,595,507\end{array}$ & 1 & 22.35 \\
\hline ZNF278 & $\begin{array}{l}\text { chr22:31,721,790- } \\
31,742,249\end{array}$ & / & 63.53 & $F C G R 2 B$ & $\begin{array}{l}\operatorname{chr} 1: 161,632,905- \\
161,648,444\end{array}$ & 1 & 21.18 \\
\hline$N F 2$ & $\begin{array}{l}\text { chr22:29,999,545- } \\
30,094,589\end{array}$ & TS & 62.35 & $C D C 73$ & $\begin{array}{l}\text { chr1:193,091,088- } \\
193,223,942\end{array}$ & TS & 21.18 \\
\hline MN1 & $\begin{array}{l}\text { chr22:28,144,265- } \\
28,197,486\end{array}$ & / & 62.35 & PTPRC & $\begin{array}{l}\text { chr1:198,608,098- } \\
198,726,605\end{array}$ & 1 & 20.00 \\
\hline CHEK2 & $\begin{array}{l}\text { chr22:29,083,731- } \\
29,137,822\end{array}$ & TS & 62.35 & MDM4 & $\begin{array}{l}\operatorname{chr} 1: 204,485,507- \\
204,527,248\end{array}$ & OG & 20.00 \\
\hline EWSR1 & $\begin{array}{l}\text { chr22:29,663,998- } \\
29,696,515\end{array}$ & / & 62.35 & $E L K 4$ & $\begin{array}{l}\text { chr1:205,566,695- } \\
205,602,000\end{array}$ & / & 20.00 \\
\hline$B C R$ & $\begin{array}{l}\text { chr22:23,522,552- } \\
23,660,224\end{array}$ & OG & 60.00 & $S L C 45 A 3$ & $\begin{array}{l}\text { chr1:205,626,981- } \\
205,649,630\end{array}$ & 1 & 20.00 \\
\hline$S M A R C B 1$ & $\begin{array}{l}\text { chr22:24,129,150- } \\
24,176,705\end{array}$ & / & 60.00 & $H L F$ & $\begin{array}{l}\text { chr17:53,342,321- } \\
53,402,426\end{array}$ & OG & 20.00 \\
\hline$M A P K 1$ & $\begin{array}{l}\text { chr22:22,113,947- } \\
22,221,970\end{array}$ & OG & 57.65 & MSI2 & $\begin{array}{l}\operatorname{chr} 17: 55,333,931- \\
55,757,299\end{array}$ & 1 & 20.00 \\
\hline CLTCL1 & $\begin{array}{l}\text { chr22:19,167,712- } \\
19,279,239\end{array}$ & TS & 55.29 & RNF43 & $\begin{array}{l}\text { chr17:56,431,038- } \\
56,494,931\end{array}$ & 1 & 20.00 \\
\hline SEPT5 & $\begin{array}{l}\text { chr22:19,704,743- } \\
19,711,102\end{array}$ & / & 55.29 & CLTC & $\begin{array}{l}\text { chr17:57,697,050- } \\
57,774,317\end{array}$ & TS & 20.00 \\
\hline LZTR1 & $\begin{array}{l}\text { chr22:21,336,558- } \\
21,353,326\end{array}$ & TS & 55.29 & PPMID & $\begin{array}{l}\text { chr17:58,677,544- } \\
58,743,640\end{array}$ & OG & 20.00 \\
\hline$C D K N 2 A$ & $\begin{array}{l}\text { chr9:21,967,751- } \\
21,975,132\end{array}$ & TS & 50.59 & BRIP1 & $\begin{array}{l}\text { chr17:59,756,547- } \\
59,940,920\end{array}$ & TS & 20.00 \\
\hline SETD2 & $\begin{array}{l}\text { chr3:47,057,898- } \\
47,205,467\end{array}$ & TS & 44.71 & $C D 79 B$ & $\begin{array}{l}\text { chr17:62,006,098- } \\
62,009,704\end{array}$ & OG & 20.00 \\
\hline$B A P 1$ & $\begin{array}{l}\text { chr3:52,435,020- } \\
52,444,121\end{array}$ & TS & 43.53 & $D D X 5$ & $\begin{array}{l}\text { chr17:62,494,374- } \\
62,502,484\end{array}$ & OG & 20.00 \\
\hline NCKIPSD & $\begin{array}{l}\operatorname{chr} 3: 48,711,278- \\
48,723,334\end{array}$ & / & 42.35 & $A X I N 2$ & $\begin{array}{l}\text { chr17:63,524,683- } \\
63,557,740\end{array}$ & TS & 20.00 \\
\hline PBRMI & $\begin{array}{l}\text { chr3:52,579,368- } \\
52,713,739\end{array}$ & TS & 42.35 & PRKARIA & $\begin{array}{l}\operatorname{chr} 17: 66,507,921- \\
66,529,570\end{array}$ & / & 20.00 \\
\hline
\end{tabular}

aThe 20 'Cancer census genes' most frequently involved in a copy number loss or gain were identified. However, as some 'Cancer census genes' showed exactly the same frequency of loss or gain, this list can contain more than 20 genes.

${ }^{b}$ Classified as an oncogene or tumor suppressor gene according to the 'Cancer census gene' list.

'When a 'Cancer census gene' was spread over multiple segments, the frequency of the segment containing the largest part of the gene was considered.

OG: oncogene; TS: tumor suppressor gene 
associations with gender, age at diagnosis (before or after the age of 60), histological diagnosis (epithelioid or non-epithelioid), survival (more or less than 36 months) or chemotherapeutic treatment before sample collection were found (Supplementary Tables 3 and 4, depicting the $p$-values for the investigated associations).

\section{DISCUSSION}

\section{Recurrent CNVs are detected in TCGA- and LP-WGS-data}

In the past, karyotype analyses and (microarraybased) comparative genomic hybridization techniques have been employed to reveal the presence of a complex and heterogeneous set of chromosomal CNVs in MPM [10-19]. However, these techniques have a limited resolution compared to highly sensitive next-generation sequencing platforms. Therefore, we performed LPWGS on genomic DNA from 21 paired tumor and normal samples to validate the results we obtained using array data from 85 MPMs, available through TCGA.

Both in the sample set from TCGA and from LPWGS, chromosomal regions exhibiting frequent copy number loss or gain were identified (Supplementary Figure 3, comparing the CNVs in both sample sets). Losses of regions on chromosomes 1 (p31.1-p13.1), 3 (p22.2-p14.2), 4 (q13.1-q35.2), 6 (q15-q27), 9 (p22.2-p21.1), 13 (q11-q22.3) and 22 (q11.1-q13.33) were found in at least $25 \%$ of MPMs, both in the TCGA-set and LP-WGS-sample set. Regions with recurrent copy number gain occurred less frequently. Nevertheless, gains were detected on chromosomes 1 (q21.2-q44), 5 (p15.33-p11), 7 (p22.3-p11.2 and q11.21-q31.33) and 17 (q21.32-q25.1) in more than $15 \%$ of MPMs in both sample sets. Next to these similarities, some differences between both sample sets could be noted. The most striking of these differences was a loss of regions on chromosomes 1 (p36.33-p36.13) and 14 (q11.1-q32.33) which was not present in our LPWGS-sample set. Moreover, some copy number gains identified in the LP-WGS-sample set on chromosomes 2 (p25.3-p22.3), 3 (q24-q29), 5 (q11.1-q35.3), 18 (p11.32-p11.21 and q11.1-q23) and 19 (p13.3-p12 and q11-q13.43) were not that frequent in the TCGA-data. These differences might be explained by the fact that the TCGA-set was significantly bigger than LP-WGS-set (i.e. 85 versus 21 MPMs). Furthermore, different techniques with different resolutions were used to identify CNVs (i.e. SNP-array and LP-WGS). Whereas the 'Affymetrix Genome-Wide SNP Array 6.0' allowed the analysis of more than 906,600 markers for single nucleotide polymorphisms and more than 946,000 markers for CNVs (inter-marker distance below 700 bases), LP-WGS achieved an average genome-wide coverage of $1.21 \mathrm{x}$.

\section{'Cancer census genes' are located in regions exhibiting recurrent $\mathrm{CNVs}$}

In order to identify potentially interesting genes within the regions exhibiting recurrent CNVs, the exact frequency of copy number loss and gain in the regions containing 'Cancer census genes' was determined, both for

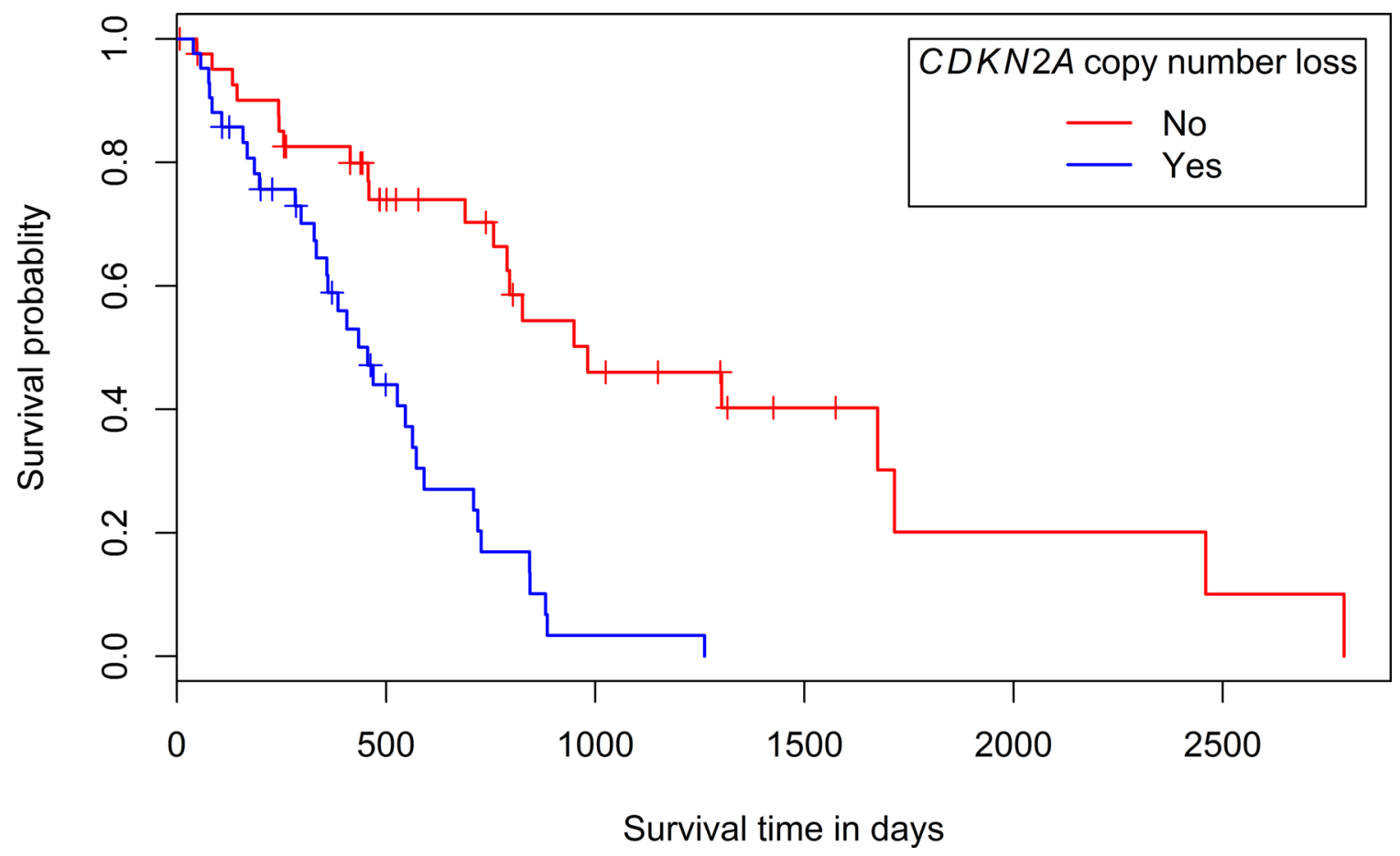

Figure 2: Kaplan-Meier plot of the overall survival according to the presence or absence of copy number loss in the chromosomal region containing $C D K N 2 A$. 
the TCGA- and LP-WGS-sample set [21]. The inactivation of the tumor suppressor genes $C D K N 2 A, N F 2$ and $B A P 1$ is well documented in MPM. Hence, it was no surprise that the regions in which these genes are located were frequently involved in a copy number loss in both sample sets. Whereas $C D K N 2 A$ was lost in $51 \%$ of TCGA-samples and $52 \%$ of in-house samples, $N F 2$ exhibited loss in $62 \%$ and $48 \%$, and BAP1 in $44 \%$ and $43 \%$ of TCGA- and inhouse samples respectively. Although these frequencies were sufficient to rank these genes in the 'top 20' list of 'Cancer census genes' most frequently involved in a copy number loss in the TCGA-sample set, this was not the case for our in-house sample set. However, given the recurrent deletion of $C D K N 2 A$ in MPM (in more than 50\% of cases in both datasets), its detection could be useful in a diagnostic and therapeutic setting. Regarding MPM- diagnosis, the use of fluorescence in situ hybridization to detect the homozygous deletion of CDKN2A proved helpful to distinguish between malignant mesothelial cells and benign reactive mesothelial cells both in pleural effusion and tissue samples [26, 27]. Regarding MPMtherapy, inactivation of $C D K N 2 A$ results in deregulation of CDK4 and CDK6, which makes MPMs good candidate responders to CDK4- and CDK6-inhibitory drugs. In hormone receptor-positive metastatic breast cancer, palbociclib, an inhibitor of CDK4 and CDK6, significantly improves progression-free survival [28]. Currently, the option of starting a phase II study with small molecule CDK-inhibitors in patients with refractory MPM is being investigated (NCT02187783). It should however be noted that also $R B 1$ was frequently involved in a copy number loss in the LP-WGS-sample set. As loss of RB1-function

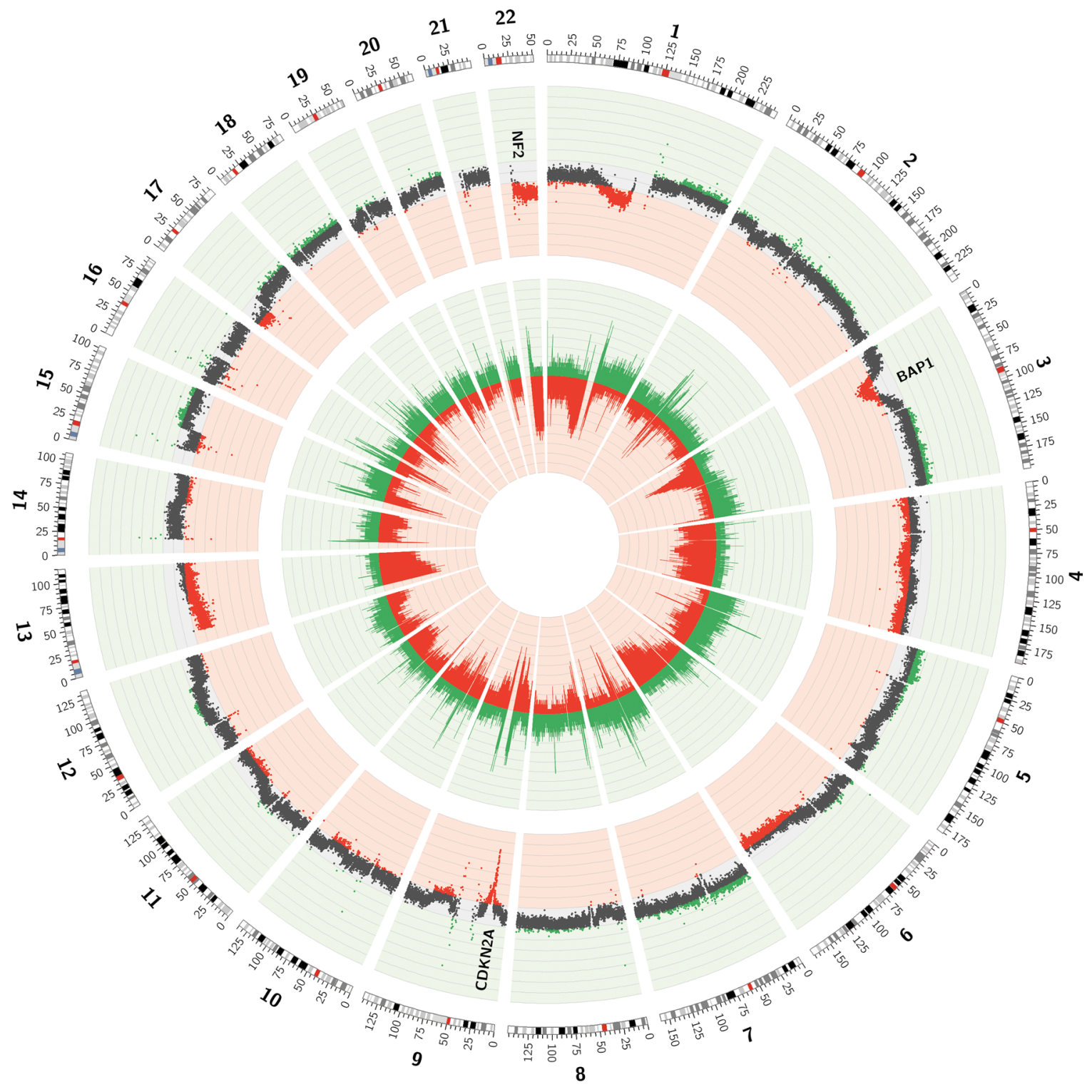

Figure 3: Circos plot of the CNVs observed in low-pass whole genome data of 21 MPMs. The inner layer represents the frequency of copy number loss (red) and gain (green) in every $50 \mathrm{~kb}$-bin. The outer layer represents the mean $\log _{2}$-ratio for every $50 \mathrm{~kb}$-bin over the 21 tumor/normal sample pairs. Mean $\log _{2}$-ratios smaller than -0.10 or bigger than 0.10 are depicted in red or green respectively. 
Table 3: 'Cancer census genes' most frequently involved in a copy number loss or gain in the LP-WGS-data

\begin{tabular}{|c|c|c|c|c|c|c|c|}
\hline \multicolumn{4}{|c|}{ 'Cancer census genes' most frequently involved in a copy number loss ${ }^{\mathrm{a}}$} & \multicolumn{4}{|c|}{ 'Cancer census genes' most frequently involved in a copy number gain ${ }^{\mathrm{a}}$} \\
\hline Gene name & Chromosome position & $\begin{array}{c}\text { OG or } \\
\text { TS }^{\mathrm{b}}\end{array}$ & $\begin{array}{c}\text { Frequency loss } \\
(\%)^{\mathrm{c}}\end{array}$ & Gene name & Chromosome position & $\begin{array}{c}\text { OG or } \\
\text { TS }^{\mathrm{b}}\end{array}$ & $\begin{array}{c}\text { Frequency } \\
\text { gain }(\%)^{\mathrm{c}}\end{array}$ \\
\hline EP300 & \begin{tabular}{|l|} 
chr22:41,488,614- \\
$41,576,081$ \\
\end{tabular} & / & 71.43 & $P M S 2$ & $\begin{array}{l}\text { chr7:6,012,870- } \\
6,048,737\end{array}$ & / & 33.33 \\
\hline SETD2 & \begin{tabular}{|l|} 
chr3:47,057,898- \\
$47,205,467$ \\
\end{tabular} & TS & 66.67 & $F C G R 2 B$ & $\begin{array}{l}\text { chr1:161,632,905- } \\
161,648,444 \\
\end{array}$ & / & 23.81 \\
\hline PBRM1 & $\begin{array}{l}\text { chr3:52,579,368- } \\
52,713,739 \\
\end{array}$ & TS & 66.67 & EIF4A2 & $\begin{array}{l}\text { chr3:186,501,361- } \\
186,507,685\end{array}$ & / & 23.81 \\
\hline ROS1 & \begin{tabular}{|l|} 
chr6:117,609,530- \\
$117,747,018$ \\
\end{tabular} & OG & 66.67 & TERT & $\begin{array}{l}\text { chr5:1,253,287- } \\
1,295,162\end{array}$ & / & 23.81 \\
\hline ZNF198 & 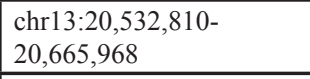 & / & 66.67 & $H N R N P A 2 B 1$ & $\begin{array}{l}\text { chr7:26,229,556- } \\
26,240,413\end{array}$ & 1 & 23.81 \\
\hline$R B 1$ & \begin{tabular}{|l|} 
chr13:48,877,883- \\
$49,056,026$ \\
\end{tabular} & TS & 66.67 & $E G F R$ & $\begin{array}{l}\text { chr7:55,086,725- } \\
55,275,031 \\
\end{array}$ & / & 23.81 \\
\hline CHEK2 & \begin{tabular}{|l|} 
chr22:29,083,731- \\
$29,137,822$ \\
\end{tabular} & TS & 66.67 & $M E T$ & $\begin{array}{l}\text { chr7:116,312,459- } \\
116,438,440 \\
\end{array}$ & OG & 23.81 \\
\hline TRIM33 & $\begin{array}{l}\text { chr1:114,935,399- } \\
115,053,781 \\
\end{array}$ & / & 61.90 & RAD21 & $\begin{array}{l}\text { chr8:117,858,173- } \\
117,887,105\end{array}$ & / & 23.81 \\
\hline CACNA1D & \begin{tabular}{|l|} 
chr3:53,529,076- \\
$53,846,492$ \\
\end{tabular} & OG & 61,90 & KLF6 & $\begin{array}{l}\text { chr10:3,818,188- } \\
3,827,473\end{array}$ & / & 23.81 \\
\hline FLT3 & \begin{tabular}{|l} 
chr13:28,577,411- \\
$28,674,729$ \\
\end{tabular} & OG & 61.90 & $N A B 2$ & $\begin{array}{l}\text { chr12:57,482,677- } \\
57,489,259\end{array}$ & OG & 23.81 \\
\hline FOXO1 & \begin{tabular}{|l|} 
chr13:41,129,801- \\
$41,240,734$ \\
\end{tabular} & $\mathrm{OG} / \mathrm{TS}$ & 61.90 & MLLT6 & $\begin{array}{l}\text { chr17:36,861,873- } \\
36,886,056\end{array}$ & / & 23.81 \\
\hline$M K L 1$ & $\begin{array}{l}\text { chr22:40,806,292- } \\
41,032,690\end{array}$ & / & 61.90 & $C I C$ & $\begin{array}{l}\text { chr19:42,788,817- } \\
42,799,949\end{array}$ & OG/TS & 23.81 \\
\hline EPS15 & \begin{tabular}{|l}
$\operatorname{chr} 1: 51,819,935-$ \\
$51,984,995$ \\
\end{tabular} & / & 57.14 & FAM131B & $\begin{array}{l}\text { chr7:143,050,493- } \\
143,059,840\end{array}$ & 1 & 19.05 \\
\hline WHSC1 & chr4:1,873,123-1,983,934 & / & 57.14 & PLAG1 & $\begin{array}{l}\text { chr8:57,073,468- } \\
57,123,859\end{array}$ & OG & 19.05 \\
\hline PTPN13 & \begin{tabular}{|l|} 
chr4:87,515,468- \\
$87,736,328$ \\
\end{tabular} & TS & 57.14 & CHCHD7 & $\begin{array}{l}\text { chr8:57,124,315- } \\
57,131,176 \\
\end{array}$ & / & 19.05 \\
\hline$R A P I G D S 1$ & \begin{tabular}{|l} 
chr4:99,182,527- \\
$99,365,012$ \\
\end{tabular} & / & 57.14 & RECQL4 & $\begin{array}{l}\text { chr8:145,736,667- } \\
145,743,210 \\
\end{array}$ & / & 19.05 \\
\hline$F B X W 7$ & \begin{tabular}{|l} 
chr4:153,242,410- \\
$153,456,185$ \\
\end{tabular} & / & 57.14 & $N U T M 2 B$ & $\begin{array}{l}\text { chr10:81,462,983- } \\
81,472,513 \\
\end{array}$ & / & 19.05 \\
\hline FAT1 & \begin{tabular}{|l|} 
chr4:187,508,937- \\
$187,644,987$ \\
\end{tabular} & TS & 57.14 & NUTM2A & $\begin{array}{l}\text { chr10:88,985,205- } \\
88,994,733 \\
\end{array}$ & 1 & 19.05 \\
\hline$N F I B$ & $\begin{array}{l}\text { chr9:14,081,842- } \\
14,314,045\end{array}$ & / & 57.14 & ETNK1 & $\begin{array}{l}\text { chr12:22,778,076- } \\
22,843,608\end{array}$ & / & 19.05 \\
\hline MLLT3 & $\begin{array}{l}\text { chr9:20,344,968- } \\
20,622,514 \\
\end{array}$ & OG & 57.14 & DICERI & $\begin{array}{l}\text { chr14:95,552,565- } \\
95,608,085\end{array}$ & TS & 19.05 \\
\hline$B R C A 2$ & $\begin{array}{l}\text { chr13:32,889,617- } \\
32,973,809\end{array}$ & TS & 57.14 & $C D 79 B$ & $\begin{array}{l}\text { chr17:62,006,098- } \\
62,009,704\end{array}$ & OG & 19.05 \\
\hline LHFP & \begin{tabular}{|l|} 
chr13:39,917,029- \\
$40,177,356$ \\
\end{tabular} & / & 57.14 & PRKARIA & $\begin{array}{l}\operatorname{chr} 17: 66,507,921- \\
66,529,570\end{array}$ & / & 19.05 \\
\hline$L C P 1$ & $\begin{array}{l}\text { chr13:46,700,058- } \\
46,756,459\end{array}$ & / & 57.14 & \multirow[t]{2}{*}{ ZNF521 } & \multirow[t]{2}{*}{$\begin{array}{l}\text { chr18:22,641,888- } \\
22,932,214\end{array}$} & \multirow[t]{2}{*}{ / } & \multirow[t]{2}{*}{19.05} \\
\hline MAPK1 & $\begin{array}{l}\text { chr22:22,113,947- } \\
22,221,970\end{array}$ & OG & 57.14 & & & & \\
\hline
\end{tabular}

aThe 20 'Cancer census genes' most frequently involved in a copy number loss or gain were identified. However, as some 'Cancer census genes' showed exactly the same frequency of loss or gain, this list can contain more than 20 genes.

${ }^{b}$ Classified as an oncogene or tumor suppressor gene according to the 'Cancer census gene' list.

'For 'Cancer census genes' smaller than $50 \mathrm{~kb}$, the frequency of copy number loss or gain in the $50 \mathrm{~kb}$-region containing at least $90 \%$ of the gene was considered. 'Cancer census genes' smaller than $50 \mathrm{~kb}$, that were not located for at least $90 \%$ in one bin, were excluded from this analysis. For the analysis of 'Cancer census genes' bigger than $50 \mathrm{~kb}$, additional bins with the exact chromosomal location of these genes were analyzed.

OG: oncogene; TS: tumor suppressor gene 
Table 4: Chromosomal regions showing copy number loss or gain in LP-WGS-dataset

\begin{tabular}{|c|c|c|c|}
\hline \multicolumn{2}{|c|}{ Copy number loss } & \multicolumn{2}{|c|}{ Copy number gain } \\
\hline Chromosome & Chromosomal region & Chromosome & Chromosomal region \\
\hline 1 & p31.1-p11.2 & \multirow{4}{*}{1} & $\mathrm{q} 25.3-\mathrm{q} 32.2$ \\
\hline 3 & p22.3-p14.1 & & $\mathrm{q} 32.3-\mathrm{q} 42.13$ \\
\hline \multirow{8}{*}{4} & p16.3-p16.1 & & $\mathrm{q} 42.13-\mathrm{q} 43$ \\
\hline & p16.1-p15.1 & & $q 43-q 44$ \\
\hline & p15.1-p12 & \multirow{8}{*}{2} & p15-p14 \\
\hline & q13.1-q25 & & p14-p13.3 \\
\hline & q26-q28.1 & & $\mathrm{q} 22.2-\mathrm{q} 22.3$ \\
\hline & q28.1-q28.3 & & $\mathrm{q} 22.3-\mathrm{q} 23.3$ \\
\hline & $\mathrm{q} 31.1-\mathrm{q} 31.21$ & & $\mathrm{q} 24.1-\mathrm{q} 24.2$ \\
\hline & $\mathrm{q} 31.21-\mathrm{q} 35.1$ & & $\mathrm{q} 31.1$ \\
\hline \multirow{3}{*}{6} & q14.3-q15 & & $\mathrm{q} 31.3-\mathrm{q} 32.1$ \\
\hline & q16.1-q21 & & $\mathrm{q} 32.1-\mathrm{q} 32.3$ \\
\hline & q21-q27 & \multirow{7}{*}{3} & $\mathrm{q} 12.1-\mathrm{q} 13.13$ \\
\hline \multirow{4}{*}{9} & $\mathrm{p} 22.2-\mathrm{p} 21.2$ & & q13.13-q13.31 \\
\hline & $\mathrm{q} 21.2-\mathrm{q} 21.31$ & & $\mathrm{q} 21.1-\mathrm{q} 21.2$ \\
\hline & $\mathrm{q} 21.31-\mathrm{q} 22.1$ & & $\mathrm{q} 21.3-\mathrm{q} 22.1$ \\
\hline & $\mathrm{q} 22.31-\mathrm{q} 22.32$ & & q24-q26.1 \\
\hline \multirow{4}{*}{10} & $\mathrm{q} 23.1$ & & $\mathrm{q} 26.1-\mathrm{q} 26.33$ \\
\hline & q23.31-q23.33 & & $\mathrm{q} 26.33-\mathrm{q} 28$ \\
\hline & $\mathrm{q} 24.2-\mathrm{q} 24.31$ & \multirow{2}{*}{5} & $\mathrm{p} 15.33-\mathrm{p} 15.2$ \\
\hline & $\mathrm{q} 25.1$ & & $\mathrm{p} 15.2-\mathrm{q} 11.1$ \\
\hline \multirow{3}{*}{11} & $\mathrm{q} 21-\mathrm{q} 22.1$ & 6 & $\mathrm{p} 25.3-\mathrm{p} 25.2$ \\
\hline & q22.3-q23.1 & \multirow{6}{*}{7} & p22.1-p13 \\
\hline & $\mathrm{q} 23.1-\mathrm{q} 23.2$ & & p13-p11.2 \\
\hline 13 & q11-q34 & & $\mathrm{q} 11.23-\mathrm{q} 21.12$ \\
\hline 17 & p13.3-p11.2 & & $\mathrm{q} 21.13-\mathrm{q} 21.3$ \\
\hline \multirow[t]{21}{*}{22} & \multirow[t]{21}{*}{ q11.1-q13.31 } & & $\mathrm{q} 31.1-\mathrm{q} 31.31$ \\
\hline & & & q32.2-q33 \\
\hline & & \multirow{3}{*}{8} & $\mathrm{q} 12.1$ \\
\hline & & & $\mathrm{q} 21.13$ \\
\hline & & & $\mathrm{q} 21.3-\mathrm{q} 22.3$ \\
\hline & & \multirow{2}{*}{12} & $\mathrm{q} 14.1-\mathrm{q} 15$ \\
\hline & & & $\mathrm{q} 15-\mathrm{q} 21.1$ \\
\hline & & \multirow{2}{*}{15} & $\mathrm{q} 21.3-\mathrm{q} 22.31$ \\
\hline & & & $\mathrm{q} 22.31-\mathrm{q} 23$ \\
\hline & & \multirow{10}{*}{18} & p11.32-p11.31 \\
\hline & & & p11.31-p11.21 \\
\hline & & & $\mathrm{q} 12.1$ \\
\hline & & & $\mathrm{q} 12.2-\mathrm{q} 12.3$ \\
\hline & & & $\mathrm{q} 12.3-\mathrm{q} 21.1$ \\
\hline & & & $\mathrm{q} 21.1-\mathrm{q} 21.2$ \\
\hline & & & $\mathrm{q} 21.2-\mathrm{q} 21.31$ \\
\hline & & & $\mathrm{q} 21.31-\mathrm{q} 21.32$ \\
\hline & & & $\mathrm{q} 21.33-\mathrm{q} 22.1$ \\
\hline & & & $\mathrm{q} 22.2-\mathrm{q} 23$ \\
\hline & & \multirow{2}{*}{20} & q13.12-q13.13 \\
\hline & & & $\mathrm{q} 13.2-\mathrm{q} 13.32$ \\
\hline
\end{tabular}


is reported to be a mechanism of resistance to CDKinhibitors, a subset of MPMs might have to be excluded from trials aiming at proving the efficacy of CDKinhibitors in MPM [29].

Both in the TCGA- and LP-WGS-sample set, other interesting cancer-associated genes were listed as being frequently involved in a copy number loss (Tables 2 and 3). Strikingly, both sample sets shared six genes in their 'top 20' list of most frequently lost 'Cancer census genes' (i.e. EP300, SETD2, PBRM1, CHEK2, MKL1 and $M A P K 1) . E P 300$, in both sample sets the 'Cancer census gene' with the highest reported frequency of copy number loss, encodes an histone acetyltransferase, important in cell proliferation and differentiation [22, 23]. EP300 has been reported to play a role in tumorigenesis, and inactivating mutations in EP300 have been described in several solid tumor types (e.g. colorectal and gastric tumors) [30]. However, not much is known about the role of EP300 in MPM. SETD2, encoding a member of the SET-domain family containing histone methyltransferases [31], and $P B R M 1$, encoding a subunit of ATP-dependent chromatin remodeling complexes [32], have been recently linked to MPM. Not only mutations, gene fusions and splice alterations were described, also frequent minute deletions were found in these genes $[19,33,34]$. Moreover, silencing of SETD2 or PBRM1 was found to increase proliferation in a mesothelioma cell line [34]. Regarding the cancer-associated genes CHEK2, encoding a cell cycle checkpoint regulator; $M K L 1$, encoding a protein amongst others involved in transducing signals from the cytoskeleton to the nucleus; and MAPK1, encoding an essential component of the MAP kinase signal transduction pathway, not much is known about their role in MPM. Yet, one study did report that a substantial amount of miRNAs, downregulated in MPM, targeted MAPK1, which might suggest that this molecule is overexpressed in MPM, in contrast to our results [35].

Although in both sample sets the frequency of copy number gain in regions containing 'Cancer census genes' was remarkably lower compared to the frequency of copy number loss, some interesting genes were among the most frequently gained ones (Tables 2 and 3). Moreover, both sample sets shared four genes in their 'top 20' list of 'Cancer census genes' most frequently involved in a copy number gain (i.e. TERT, FCGR2B, CD79B and PRKAR1A). TERT, the 'Cancer census gene' exhibiting the most frequent copy number gain in the TCGAset, encodes the catalytic component of the telomerase enzyme. Telomerase expression is normally repressed in postnatal somatic cells resulting in progressive shortening of the telomeres. However, deregulation of telomerase expression in somatic cells can contribute to a replicative immortality, which is one of the 'Hallmarks of Cancer' [36]. In line with this function, TERT expression was detected in $99 \%$ of MPMs using immunohistochemistry and in situ hybridization [37]. Furthermore, TERT mRNA was found to be upregulated in MPM. Nonetheless, this upregulation was reported to be the result of mutations in the TERT promoter and not of gene copy number amplification [38]. Regarding the cancer-associated genes $F C G R 2 B$, encoding a low affinity receptor for the $\mathrm{Fc}-$ region of immunoglobulin gamma complexes; $C D 79 B$, encoding the immunoglobulin beta protein which is necessary for functioning of the B-cell antigen receptor; and PRKAR1A, encoding one of the regulatory subunits of the cAMP-dependent protein kinase, not much is known about their role in MPM. Strikingly, PMS2, the 'Cancer census gene' most frequently involved in a copy number gain in the LP-WGS-set, was not ranked among the most frequently gained genes in the TCGA-set. As this gene encodes a component of the DNA mismatch repair system [25], one would not expect a copy number gain of the region containing this gene. However, overexpression of PMS2 was previously reported to confer genetic instability and DNA-damage tolerance in prostate cancer $[39,40]$.

Regarding these results, it should be noted that the 'Cancer census genes' that are most frequently involved in a copy number loss or gain, are clustered in certain regions (Tables 2 and 3). For example, a substantial amount of the most lost genes in the TCGA-dataset are located on chromosome 22. As chromosome 22 is almost entirely lost in more than $60 \%$ of MPMs in this dataset, this is no surprise. Obviously, not all listed genes will be equally important in MPM-tumorigenesis, and some genes will only be listed as they are in the proximity of more important ones. This might explain why even some oncogenes (e.g. MAPK1) pop up. Only further functional studies can elucidate the role of each of the listed genes in the pathogenesis of MPM.

\section{$C D K N 2 A$ loss is associated with a shorter overall survival}

In the LP-WGS-set, no statistically significant associations between any of the investigated clinicopathological parameters and the presence of copy number loss or gain in regions with selected 'Cancer census genes' were found (Supplementary Tables 3 and 4, depicting the $p$-values for the investigated associations). In the TCGA-set however, a statistically significant association was found between an overall survival shorter than 36 months and the presence of copy number loss in the chromosomal segment containing $C D K N 2 A$, which was confirmed by a univariate survival analysis (Supplementary Table 1 and Figure 2). Differences in prognosis according to $C D K N 2 A$ deletion status and CDKN2A (p16ink4a/p14ARF) protein expression were previously reported [41-45]. In several reports, a statistically significant survival advantage was found for patients with tumors without $C D K N 2 A$ homozygous deletion $[41,42,45]$. Moreover, in studies by Dacic et al. and Kobayashi et al., loss of CDKN2A (p16ink4a) protein 
expression, as detected by immunohistochemistry, was shown to be associated with a poor prognosis. Whereas Dacic et al. also reported significant differences in survival according to the homozygous deletion status of $C D K N 2 A$, this was not mirrored by Kobayashi et al. [42, 43]. In a study by Walter et al., a survival difference was seen between patients with a low CDKN2A (p14ARF) mRNA-expression and patients with a high expression. Nevertheless, the association between overall survival and CDKN2A (p14ARF) mRNA-expression did not reach statistical significance [44]. Given the limited therapeutic options for MPM-patients, their modest benefit and sometimes substantial toxicity, identifying patients with a particularly poor prognosis can be beneficial. Hence, the potential utility of $C D K N 2 A$ deletion in a prognostic setting holds promise for the future.

\section{MATERIALS AND METHODS}

\section{TCGA-data collection}

TCGA is a joint effort of the National Cancer Institute (NCI) and the National Human Genome Research Institute (NHGRI), that has generated comprehensive maps of the key genomic changes in 33 types of cancer (http://cancergenome.nih.gov/). Regarding MPM, TCGA holds data of 87 patients, including segmented copy number data (TCGA level 3 data, hg19.seg-files). For the latter, the original data files were generated using the 'Affymetrix Genome-Wide SNP Array 6.0' (Thermo Fisher Scientific, Waltham, MA, USA), and the files were analyzed using the 'CopyNumberInferencePipeline' in 'GenePattern' [46]. In each of the resulting files, segment means were normalized against a panel of several thousands of blood normal samples. Patient characteristics of the TCGA-patients are summarized in Table 1. Two patients in the TCGA MPM-cohort received neo-adjuvant treatment, whereas all others did not. Therefore, we chose to exclude these two patients from all analyses.

\section{Copy number profiling of TCGA-data}

In order to identify recurrent copy number differences in the MPM-samples of which segmented copy number data were available through TCGA, frequencies of copy number loss and gain were calculated. In this respect, regions with segment means smaller or equal than -0.25 were considered as losses and regions with segment means bigger or equal than 0.25 were considered as gains. Using the 'Multi-intersect tool' from 'BEDtools' [20], chromosomal regions with recurrent copy number loss or gain in the 85 MPMs were identified, after which frequencies were calculated. In order to identify potentially interesting genes within regions exhibiting recurrent CNVs, the frequency of copy number loss and gain specifically in the regions containing 'Cancer census genes' was determined. The 'Cancer census genes' are genes with substantial published evidence in oncology. This list, containing 609 genes at the time of first analysis (accessed in November 2016), is regularly updated by the COSMIC team and can be found on their website [21].

\section{Patient samples collection and preparation}

In order to validate the results obtained using TCGA-data, LP-WGS was performed on an independent MPM-cohort. This study was conducted with the approval of the ethical committee of the Antwerp University Hospital and the University of Antwerp (Reference numbers 14/8/73 \& 16/23/248). Twenty-one MPM- and matched normal samples were obtained from the tumor bank of the Antwerp University Hospital (Biobank@UZA, Antwerp, Belgium; ID: BE71030031000, Belgian Virtual Tumorbank funded by the National Cancer Plan) and from the tissue bank of the Erasmus University Medical Center Rotterdam. Patient characteristics are summarized in Table 1. Non-tumor material consisted of cryopreserved blood lymphocytes, collected before or after surgery. When matched blood samples were not available, healthy lung or pleura tissue, removed during resection, was used. All tissue samples were collected in the operating room, immediately snap-frozen in liquid nitrogen and stored at $-80^{\circ} \mathrm{C}$. Diagnosis and tumor content were confirmed by histological examination of hematoxylin-eosin-stained $5 \mu \mathrm{m}$-sections. Histology of the tumor samples included epithelioid $(N=18)$, biphasic $(N=2)$ and epithelioid/ desmoplastic $(N=1)$. DNA was extracted from each of the blood samples and from fifteen $10 \mu \mathrm{m}$-sections per tissue sample using the 'QIAamp DNA Mini Kit' (Qiagen, Hilden, Germany, Cat. No. 51304), according to the manufacturer's instructions.

\section{Copy number profiling of LP-WGS-data}

Genomic DNA was fragmented using a Covaris instrument (Covaris, Woburn, MA, USA) and sequencing libraries were generated using the 'KAPA Library Preparation Kit' (Roche, Basel, Switzerland, Cat. No. KK8230). Next, sample libraries were sequenced on an 'Illumina HiSeq 1500 platform' (Illumina, San Diego, CA, USA) in high output mode, generating $2 \times 100$ bp pairedend reads. This resulted in an average coverage of $1.21 \mathrm{x}$, which enabled the detection of structural variants.

Sequencing reads were adapter trimmed and mapped to the UCSC human genome (GRCh37/hg19). The presence of CNVs in the samples was analyzed using inhouse developed analysis pipelines. The algorithm divides the genome into non-overlapping $50 \mathrm{~kb}$-bins and counts all mapped sequencing reads for each tumor and normal sample within each bin. After correction of read counts for local GC-content using lowess normalization, $\log _{2}$-ratios were calculated for every tumor and normal sample pair. 
In order to identify recurrent copy number differences between tumor and normal samples, frequencies of copy number loss and gain were calculated for each of the $50 \mathrm{~kb}$-bins. We used a $\log _{2}$ ratio threshold of -0.25 for chromosomal losses and 0.25 for copy number gains. Similar as for the TCGA-data, the frequency of copy number loss and gain specifically in the regions containing 'Cancer census genes' was assessed [21]. For 'Cancer census genes' smaller than $50 \mathrm{~kb}$, the frequency of copy number loss and gain in the $50 \mathrm{~kb}$-region containing at least $90 \%$ of the gene was considered. 'Cancer census genes' smaller than 50 $\mathrm{kb}$, that were not located for at least $90 \%$ in one bin, were excluded from this analysis. To enable the analysis of 'Cancer census genes' bigger than $50 \mathrm{~kb}$, additional bins with the exact chromosomal location of these genes were analyzed. Next to this frequency-based approach, the mean $\log _{2}$-ratio for each $50 \mathrm{~kb}$-bin over the 21 sample pairs was determined. This is a novel approach enabling the identification of recurrent copy number differences between tumor and normal samples. Within each of the $50 \mathrm{~kb}$-bins, a one-sample $t$-test was carried out, testing the null hypothesis that the mean $\log _{2}$-ratio within this bin equals 0 . If all null hypotheses are true across all bins tested, it is expected that the $p$-values of these tests follow a uniform distribution with boundaries 0 and 1. This latter hypothesis was tested using the K-S test. One K-S test was carried out per sliding window of 50 $p$-values (coming from the one-sample $t$-test). The step width between the sliding windows was set to 25 .

\section{Statistical analysis}

To identify associations between clinicopathological parameters on the one hand and the presence of copy number loss or gain in regions containing selected genes on the other hand, a Pearson's Chi-squared test with Yates' continuity correction was performed. In case more than $20 \%$ of the cells had an expected count below five, a Fisher's Exact test was used. Segment means (TCGAdata) and $\log _{2}$-ratios (LP-WGS-data) smaller or equal than -0.25 were considered as losses and values bigger or equal than 0.25 were considered as gains.

Parameters such as the presence of copy number loss or gain in regions containing selected genes, gender and histological subtype, were tested for association with overall survival using the log-rank test. In this respect, overall survival was defined as the time from initial pathologic diagnosis to the date of death or last follow-up. Survival curves were plotted using the method of Kaplan and Meier.

All $p$-values were based on a two-sided hypothesis, with $p$-values smaller or equal than 0.05 considered statistically significant. Decimal values were rounded to the nearest digit. Statistical analyses were carried out using the statistical software ' $\mathrm{R}$ ' version 2.3.1. [47].

\section{CONCLUSIONS}

Recurrent copy number losses and gains were identified in the TCGA-set and confirmed in an inhouse sample set using LP-WGS. These CNVs occurred in regions harboring cancer-associated genes that are potentially useful in a diagnostic, therapeutic and prognostic setting.

\section{Abbreviations}

CNV: Copy number variation; K-S: KolmogorovSmirnov; LP-WGS: Low-pass whole genome sequencing; MPM: Malignant pleural mesothelioma; TCGA: The Cancer Genome Atlas.

\section{Author contributions}

M.H. designed and performed the experiments, analyzed the data and wrote the manuscript. G.V. developed the analysis pipeline used to analyze the LPWGS-data. M.B. and E.F. assisted with the bioinformatic and statistical data analysis. P.P. and R.C. helped in obtaining the human biological material and P.P. analyzed all hematoxylin-eosin-stained slides. G.V.C., A.S., P.P., J.V.M. and K.O.D.B. supervised the research, designed the experiments and contributed in the interpretation of the data. All authors contributed to the final version of the manuscript.

\section{ACKNOWLEDGMENTS}

The results published here are in part based upon data generated by the TCGA Research Network: http:// cancergenome.nih.gov/. The human biological material used in this publication was provided by Biobank@ UZA (Antwerp University Hospital, Belgium; ID: BE71030031000, funded by the Belgian National Cancer Plan) and by the Erasmus MC Tissue Bank (Erasmus University Medical Center, Rotterdam, The Netherlands).

\section{CONFLICTS OF INTEREST}

The authors declare no potential conflicts of interest.

\section{FUNDING}

This work was realized with an Emmanuel van der Schueren research grant from the Flemish League against Cancer, grant 2016/818 from the Belgian Foundation against Cancer and a grant from the Lung Cancer Group of the European Organisation for Research and Treatment of Cancer (EORTC, trials 08031 (PI: PE Van Schil) and 08052 (PI: ME O'Brien)). 


\section{REFERENCES}

1. Travis W, Brambilla E, Muller-Hermelink H, Harris C. World Health Organization Classification of Tumours. Pathology and Genetics of Tumours of the Lung, Pleura, Thymus and Heart. Lyon: IARC Press; 2004.

2. Carbone M, Kratzke RA, Testa JR. The pathogenesis of mesothelioma. Semin Oncol. 2002; 29:2-17.

3. Robinson BW, Lake RA. Advances in malignant mesothelioma. N Engl J Med. 2005; 353:1591-603.

4. LaDou J, Castleman B, Frank A, Gochfeld M, Greenberg M, Huff J, Joshi TK, Landrigan PJ, Lemen R, Myers J, Soffritti M, Soskolne CL, Takahashi K, et al. The case for a global ban on asbestos. Environ Health Perspect. 2010; 118:897-901.

5. van Meerbeeck JP, Scherpereel A, Surmont VF, Baas P. Malignant pleural mesothelioma: the standard of care and challenges for future management. Crit Rev Oncol Hematol. 2011; 78:92-111.

6. Vogelzang NJ, Rusthoven JJ, Symanowski J, Denham C, Kaukel E, Ruffie P, Gatzemeier U, Boyer M, Emri S, Manegold C, Niyikiza C, Paoletti P. Phase III study of pemetrexed in combination with cisplatin versus cisplatin alone in patients with malignant pleural mesothelioma. J Clin Oncol. 2003; 21:2636-44.

7. Prins JB, Williamson KA, Kamp MM, Van Hezik EJ, Van der Kwast TH, Hagemeijer A, Versnel MA. The gene for the cyclin-dependent-kinase-4 inhibitor, CDKN2A, is preferentially deleted in malignant mesothelioma. Int $\mathrm{J}$ Cancer. 1998; 75:649-53.

8. Sekido Y, Pass HI, Bader S, Mew DJ, Christman MF, Gazdar AF, Minna JD. Neurofibromatosis type 2 (NF2) gene is somatically mutated in mesothelioma but not in lung cancer. Cancer Res. 1995; 55:1227-31.

9. Bott M, Brevet M, Taylor BS, Shimizu S, Ito T, Wang L, Creaney J, Lake RA, Zakowski MF, Reva B, Sander C, Delsite R, Powell S, et al. The nuclear deubiquitinase BAP1 is commonly inactivated by somatic mutations and 3p21.1 losses in malignant pleural mesothelioma. Nat Genet. 2011; 43:668-72.

10. Tiainen M, Tammilehto L, Rautonen J, Tuomi T, Mattson K, Knuutila S. Chromosomal abnormalities and their correlations with asbestos exposure and survival in patients with mesothelioma. Br J Cancer. 1989; 60:618-26.

11. Bjorkqvist AM, Tammilehto L, Anttila S, Mattson K, Knuutila S. Recurrent DNA copy number changes in 1q, 4q, $6 \mathrm{q}, 9 \mathrm{p}, 13 \mathrm{q}, 14 \mathrm{q}$ and $22 \mathrm{q}$ detected by comparative genomic hybridization in malignant mesothelioma. $\mathrm{Br} \mathrm{J}$ Cancer. 1997; 75:523-7.

12. Bjorkqvist AM, Tammilehto L, Nordling S, Nurminen M, Anttila S, Mattson K, Knuutila S. Comparison of DNA copy number changes in malignant mesothelioma, adenocarcinoma and large-cell anaplastic carcinoma of the lung. Br J Cancer. 1998; 77:260-9.

13. Balsara BR, Bell DW, Sonoda G, De Rienzo A, du Manoir S, Jhanwar SC, Testa JR. Comparative genomic hybridization and loss of heterozygosity analyses identify a common region of deletion at 15q11.1-15 in human malignant mesothelioma. Cancer Res. 1999; 59:450-4.

14. Krismann M, Muller KM, Jaworska M, Johnen G. Molecular cytogenetic differences between histological subtypes of malignant mesotheliomas: DNA cytometry and comparative genomic hybridization of 90 cases. J Pathol. 2002; 197:363-71.

15. Taniguchi T, Karnan S, Fukui T, Yokoyama T, Tagawa H, Yokoi K, Ueda Y, Mitsudomi T, Horio Y, Hida T, Yatabe Y, Seto M, Sekido Y. Genomic profiling of malignant pleural mesothelioma with array-based comparative genomic hybridization shows frequent non-random chromosomal alteration regions including JUN amplification on $1 \mathrm{p} 32$. Cancer Sci. 2007; 98:438-46.

16. Ivanov SV, Miller J, Lucito R, Tang C, Ivanova AV, Pei J, Carbone M, Cruz C, Beck A, Webb C, Nonaka D, Testa JR, Pass HI. Genomic events associated with progression of pleural malignant mesothelioma. Int J Cancer. 2009; 124:589-99.

17. Cheung M, Pei J, Pei Y, Jhanwar SC, Pass HI, Testa JR. The promyelocytic leukemia zinc-finger gene, PLZF, is frequently downregulated in malignant mesothelioma cells and contributes to cell survival. Oncogene. 2010; 29:1633-40.

18. Borczuk AC, Pei J, Taub RN, Levy B, Nahum O, Chen J, Chen K, Testa JR. Genome-wide analysis of abdominal and pleural malignant mesothelioma with DNA arrays reveals both common and distinct regions of copy number alteration. Cancer Biol Ther. 2016; 17:328-35.

19. Bueno R, Stawiski EW, Goldstein LD, Durinck S, De Rienzo A, Modrusan Z, Gnad F, Nguyen TT, Jaiswal BS, Chirieac LR, Sciaranghella D, Dao N, Gustafson CE, et al. Comprehensive genomic analysis of malignant pleural mesothelioma identifies recurrent mutations, gene fusions and splicing alterations. Nat Genet. 2016; 48:407-16.

20. Quinlan AR, Hall IM. BEDTools: a flexible suite of utilities for comparing genomic features. Bioinformatics. 2010; 26:841-2.

21. Futreal PA, Coin L, Marshall M, Down T, Hubbard T, Wooster R, Rahman N, Stratton MR. A census of human cancer genes. Nat Rev Cancer. 2004; 4:177-83.

22. Ogryzko VV, Schiltz RL, Russanova V, Howard BH, Nakatani Y. The transcriptional coactivators p300 and CBP are histone acetyltransferases. Cell. 1996; 87:953-9.

23. Kawasaki H, Eckner R, Yao TP, Taira K, Chiu R, Livingston DM, Yokoyama KK. Distinct roles of the co-activators p300 and CBP in retinoic-acid-induced F9-cell differentiation. Nature. 1998; 393:284-9.

24. Weinrich SL, Pruzan R, Ma L, Ouellette M, Tesmer VM, Holt SE, Bodnar AG, Lichtsteiner S, Kim NW, Trager JB, Taylor $\mathrm{RD}$, Carlos R, Andrews WH, et al. Reconstitution of human telomerase with the template RNA component hTR and the catalytic protein subunit hTRT. Nat Genet. 1997; 17:498-502.

25. Li GM. Mechanisms and functions of DNA mismatch repair. Cell Res. 2008; 18:85-98. 
26. Illei PB, Ladanyi M, Rusch VW, Zakowski MF. The use of CDKN2A deletion as a diagnostic marker for malignant mesothelioma in body cavity effusions. Cancer. 2003; 99:51-6.

27. Wu D, Hiroshima K, Matsumoto S, Nabeshima K, Yusa T, Ozaki D, Fujino M, Yamakawa H, Nakatani Y, Tada Y, Shimada H, Tagawa M. Diagnostic usefulness of p16/ CDKN2A FISH in distinguishing between sarcomatoid mesothelioma and fibrous pleuritis. Am J Clin Pathol. 2013; 139:39-46.

28. Turner NC, Ro J, André F, Loi S, Verma S, Iwata H, Harbeck N, Loibl S, Huang Bartlett C, Zhang K, Giorgetti C, Randolph S, Koehler M, Cristofanilli M, and PALOMA3 Study Group. Palbociclib in Hormone-Receptor-Positive Advanced Breast Cancer. N Engl J Med. 2015; 373:209-19.

29. O'Leary B, Finn RS, Turner NC. Treating cancer with selective CDK4/6 inhibitors. Nat Rev Clin Oncol. 2016; 13:417-30.

30. Iyer NG, Ozdag H, Caldas C. p300/CBP and cancer. Oncogene. 2004; 23:4225-31.

31. Qian C, Zhou MM. SET domain protein lysine methyltransferases: Structure, specificity and catalysis. Cell Mol Life Sci. 2006; 63:2755-63.

32. Thompson M. Polybromo-1: the chromatin targeting subunit of the PBAF complex. Biochimie. 2009; 91:309-19.

33. Yoshikawa Y, Sato A, Tsujimura T, Otsuki T, Fukuoka K, Hasegawa S, Nakano T, Hashimoto-Tamaoki T. Biallelic germline and somatic mutations in malignant mesothelioma: multiple mutations in transcription regulators including mSWI/SNF genes. Int J Cancer. 2015; 136:560-71.

34. Yoshikawa Y, Emi M, Hashimoto-Tamaoki T, Ohmuraya M, Sato A, Tsujimura T, Hasegawa S, Nakano T, Nasu M, Pastorino S, Szymiczek A, Bononi A, Tanji M, et al. High-density array-CGH with targeted NGS unmask multiple noncontiguous minute deletions on chromosome 3p21 in mesothelioma. Proc Natl Acad Sci U S A. 2016; 113:13432-7.

35. Ramirez-Salazar EG, Salinas-Silva LC, Vazquez-Manriquez ME, Gayosso-Gomez LV, Negrete-Garcia MC, RamirezRodriguez SL, Chavez R, Zenteno E, Santillan P, KellyGarcia J, Ortiz-Quintero B. Analysis of microRNA expression signatures in malignant pleural mesothelioma, pleural inflammation, and atypical mesothelial hyperplasia reveals common predictive tumorigenesis-related targets. Exp Mol Pathol. 2014; 97:375-85.

36. Hanahan D, Weinberg RA. Hallmarks of cancer: the next generation. Cell. 2011; 144:646-74.

37. Kumaki F, Kawai T, Churg A, Galateau-Salle FB, Hasleton P, Henderson D, Roggli V, Travis WD, Cagle PT, Ferrans VJ. Expression of telomerase reverse transcriptase (TERT) in malignant mesotheliomas. Am J Surg Pathol. 2002; 26:365-70.

38. Tallet A, Nault JC, Renier A, Hysi I, Galateau-Salle F, Cazes A, Copin MC, Hofman P, Andujar P, Le Pimpec-Barthes F,
Zucman-Rossi J, Jaurand MC, Jean D. Overexpression and promoter mutation of the TERT gene in malignant pleural mesothelioma. Oncogene. 2014; 33:3748-52.

39. Norris AM, Woodruff RD, D'Agostino RB Jr, Clodfelter JE, Scarpinato KD. Elevated levels of the mismatch repair protein PMS2 are associated with prostate cancer. Prostate. 2007; 67:214-25.

40. Wilczak W, Rashed S, Hube-Magg C, Kluth M, Simon R, Buscheck F, Clauditz TS, Grupp K, Minner S, Tsourlakis MC, Moller-Koop C, Graefen M, Adam M, et al. Upregulation of mismatch repair genes MSH6, PMS2 and MLH1 parallels development of genetic instability and is linked to tumor aggressiveness and early PSA recurrence in prostate cancer. Carcinogenesis. 2017; 38:19-27.

41. Lopez-Rios F, Chuai S, Flores R, Shimizu S, Ohno T, Wakahara K, Illei PB, Hussain S, Krug L, Zakowski MF, Rusch V, Olshen AB, Ladanyi M. Global gene expression profiling of pleural mesotheliomas: overexpression of aurora kinases and P16/CDKN2A deletion as prognostic factors and critical evaluation of microarray-based prognostic prediction. Cancer Res. 2006; 66:2970-9.

42. Dacic S, Kothmaier H, Land S, Shuai Y, Halbwedl I, Morbini P, Murer B, Comin C, Galateau-Salle F, Demirag F, Zeren H, Attanoos R, Gibbs A, et al. Prognostic significance of p16/cdkn2a loss in pleural malignant mesotheliomas. Virchows Arch. 2008; 453:627-35.

43. Kobayashi N, Toyooka S, Yanai H, Soh J, Fujimoto N, Yamamoto H, Ichihara S, Kimura K, Ichimura K, Sano Y, Kishimoto T, Date H. Frequent p16 inactivation by homozygous deletion or methylation is associated with a poor prognosis in Japanese patients with pleural mesothelioma. Lung Cancer. 2008; 62:120-5.

44. Walter RF, Mairinger FD, Ting S, Vollbrecht C, Mairinger T, Theegarten D, Christoph DC, Schmid KW, Wohlschlaeger J. MDM2 is an important prognostic and predictive factor for platin-pemetrexed therapy in malignant pleural mesotheliomas and deregulation of P14/ARF (encoded by CDKN2A) seems to contribute to an MDM2-driven inactivation of P53. Br J Cancer. 2015; 112:883-90.

45. Hamasaki M, Matsumoto S, Abe S, Hamatake D, Kamei T, Hiroshima K, Kawahara K, Sato A, Tsujimura T, Nakatani Y, Yoshida Y, Iwasaki A, Nabeshima K. Low homozygous/ high heterozygous deletion status by p16 FISH correlates with a better prognostic group than high homozygous deletion status in malignant pleural mesothelioma. Lung Cancer. 2016; 99:155-61.

46. Reich M, Liefeld T, Gould J, Lerner J, Tamayo P, Mesirov JP. GenePattern 2.0. Nat Genet. 2006; 38:500-1.

47. R Core Team. R: A language and environment for statistical computing. R Foundation for Statistical Computing, Vienna, Austria. 2015. 